Ann. Biol. anim. Bioch. Biophys., 1977, 17 (2), 237-242.

\title{
Mélange fixateur à base d'acide tannique Application à l'immunocytologie
}

\author{
par E. KOLODZIEJCZYK
}

Déparfement de Biologie Humaine, Faculté de Médecine, 37000 Tours

Station de Physiologie de la Reproduction, I. N. R. A., Nouzilly,

37380 Monnaie

Summary. A tannic acid fixative mixture for use in immunocytology.

Tannic acid and formaldehyde differently associated as fixatives are used by perfusion for immunocytology.

A postfixation precipitating tannic acid preserves somatostatin in tissues while they are dehydrated and embedded in paraffin. This technique permits immunocytological study of cellular systems synthesizing the neuropeptide.

\section{Introduction.}

Les fixateurs aldéhydiques ont permis la mise en évidence immunocytologique des hormones hypothalamiques, somatostatine (SRIF) et LH-RF, au sein d'axones dans l'éminence médiane ef l'hypothalamus (Dubois ef al., 1974 ; Leonardelli et al., 1973). Le Bouin Hollande sublimé n'a pas permis entre nos mains, d'immobiliser la somatostatine au sein des pericaryons qui l'élaborent, chez le rat.

L'hypothèse selon laquelle la somatostatine, tétradécapeptide très soluble, seraił sous forme libre ou faiblement liée au sein de ces péricaryons, nous a conduit à concevoir un mélange fixateur susceptible d'immobiliser ce peptide en l'insolubilisant tant au cours de la déshydratation qu'au cours de l'inclusion. Nous avons porté notre choix sur l'acide tannique, parce qu'il présente la propriété de précipiter les protéines ef les peptones de leurs solutions aqueuses (Mizuhira ef Futaesaku, 1972).

Dans cette note, nous envisageons les propriétés de l'acide tannique vis-à-vis des peptones et des peptides que nous étudions : somatostatine et LH-RF.

\section{Matériel et méthodes.}

La recherche a comporté deux étapes :

I. - Ełude in vitro des propriétés précipitantes de l'acide tannique.

II. - Application des résultats obtenus in vitro à la fixation de matériel préparé en vue d'études histologiques. 


\section{Matériel.}

\section{I. - Etude in vitro}

L'acide tannique (Tannin gepulvert rein Art. 773 Merck) a été utilisé à la concentration finale de 4 p. 100 dans l'eau distillée.

Pour l'étude de la précipitation des peptides, nous avons eu recours à de la somatostatine cyclique de synthèse et à du LH-RF de synthèse, marqués à l'iode 125 selon la technique de Hunter et Greenwood.

Action précipitante de l'acide tannique vis-à-vis des peptones et des peptides.

\section{a) Peptones.}

Un mélange, volume à volume, d'une solution d'acide tannique à 8 p. 100 dans l'eau distillée et de peptones à la concentration de 1 p. 100 dans l'eau distillée, donne spontanément naissance à un précipité insoluble.

La réaction est visible après centrifugation pour des concentrations finales de peptones allant jusqu'à $1 / 5000$.

b) Peptides.

L'acide tannique, quelle que soit sa concentration, ne précipite ni le LH-RF ni la somatostatine marqués. Néanmoins, la formation de complexes (A. tannique-peptide) non précipitants ne peut être exclue.

La formation de ces complexes est visualisée par leur coprécipitation en présence de corps chimiques divers.

Coprécipitation des complexes [A. tannique-peptides].

L'existence d'une liaison [A. tannique-peptide] peuł être révélée par trois procédés :

a) coprécipitation par la soude normale.

b) coprécipitation par le sulfate de cuivre $\left(\mathrm{CuSO}_{4}: 3\right.$ p. 100) en solution dans du tampon acétate de sodium-acide acétique 0,4M, pH 6,5.

c) coprécipitation par le sulfate de cuivre en solution dans du tris (hydroxyméthyl) aminométhane (solution aqueuse à 2 p. 100), jusqu'à obtention d'une liqueur de Schweiłzer.

\section{Résultats.}

L'addition au mélange [A. tannique-somatostatine ou LH-RF marqués] d'une des trois solutions précédentes entraîne la formation d'un précipité. La mesure de la radioactivité du surnageant et du précipité après centrifugation a montré que 80 p. 100 environ de la radioactivité se retrouve dans le culot. Les lavages successifs du précipité à l'eau distillée ne libèrent pas de quantité notable de la radioactivité initiale.

De même, les lavages du précipité par des solutions successives d'alcool absolu ne modifient pas de façon significative la radioactivité.

II apparaît donc que la somatostatine et le LH-RF ont été immobilisés de façon irréversible. 


\section{II. - Etudes histologiques}

Elles ont été menées chez le rat blanc (Wistar).

Le principe de la fixation est le suivant :

Première étape : Destruction de la perméabilité sélective des membranes biologiques par une aldéhyde et diffusion de l'acide tannique dans les cellules. étape.

Deuxième étape : Précipitation des complexes formés au cours de la première

Deux méthodes ont été utilisées quant à l'emploi de l'ion cuivre.

\section{A) Méthode dite en deux temps.}

La deuxième étape est réalisée in vitro au cours d'une post-fixatlon par Immersion. Technique.

A. - Perfusions.

- Lavage du lit vasculaire au sérum physiologique hépariné (5 $000 \mathrm{~V} . \mathrm{I} . / 1000 \mathrm{ml})$ et procainé $(0,2$ p. 100), jusqu'à ce que le liquide ressorte tout à fait clair.

- Fixation par le mélange suivant (volume à volume) :

Solution A : Acide tannique 8 p. 100 dans du tampon acétate de sodium-acide acétique $0,4 \mathrm{M}, \mathrm{pH} 6,5$.

Solution B : Formaldéhyde à 12 p. 100 dans l'eau distillée (concentration réelle; soif : $40 \mathrm{ml}$ de la solution commerciale de formaldéhyde pour $100 \mathrm{ml}$ de la solution finale).

Le $\mathrm{pH}$ final est de 5,5.

B. - Post-fixation. - L'hypothalamus prélevé immédiatement après la perfusion est immergé dans le mélange volume à volume suivant :

- 1 volume de solution A : Sulfate de cuivre à 6 p. 100 dans du tampon. Acétate de sodium-acide acétique : $0,4 \mathrm{M}, \mathrm{pH} 6,5$.

- 1 volume de solution $B$ : Formaldéhyde à 12 p. 100 dans l'eau distillée (concentration réelle):

La durée de la post-fixation est de 24 à 48 h, en fonction du volume de la pièce.

\section{B) Méthode dite en un temps.}

Cette méthode vise à associer l'acide tannique et le sulfate de cuivre au sein d'un même mélange, au cours d'une perfusion.

Technique.

A. - Perfusions.

- Lavage du lit vasculaire selon les mêmes modalités que précédemment.

- Perfusion par le mélange fixateur suivant (volume à volume) : 
- 1 volume de solution $A$ : Sulfate de cuivre à 6 p. 100 dans une solution aqueuse à 8 p. 100 d'acide tannique.

- 1 volume de solution $B$ : Formaldéhyde à 12 p. 100 dans l'eau distillée (concentration réelle). Le $\mathrm{pH}$ de ce mélange est de 3 environ.

$150 \mathrm{ml}$ de ce mélange sont perfusés en $5 \mathrm{mn}$ environ.

B. - Post-fixation. - Elle peut être de trois types :

1) Formaldéhyde à 6 p. 100 dans du sérum physiologique (concentration réelle).

2) Mélange, volume à volume :

- d'une solution de formaldéhyde à 12 p. 100 dans l'eau distillée (concentration réelle),

- et d'une solution de tampon acide acétique-acétate de sodium :0,4M, $\mathrm{pH} 6,5$.

3) Formaldéhyde à 6 p. 100 (concentration réelle) dans une solution de tris (hydroxyméthyl) aminométhane (solution à 2 p. 100).

\section{C) Résultats.}

- Somatostatine.

L'application de ces techniques a permis la mise en évidence de deux systèmes élaborateurs de somatostatine dans l'hypothalamus du rat.

Ces résultats ont déjà fait l'objet d'une publication (Dubois et Kolodziejczyk, 1975). Nous en résumons les points essentiels :

Les péricaryons élaborateurs de somatostatine sont répartis en deux groupes morphologiquement ef topographiquement différents :

- Un système magnocellulaire neurophysine positif, représenté par des cellules du noyau supra-optique, des cellules du noyau paraventriculaire, des îlots hypothalamiques épars.

- Un système parvocellulaire, neurophysine négatif, dans la région du récessus supra-optique du troisième ventricule.

- LH-RF.

Il ne nous a pas été possible de mettre en évidence des péricaryons élaborateurs de LH-RF chez le rat, après application de ces méthodes de fixation.

Cependant le contenu en LH-RF des terminaisons axonales de l'éminence médiane et de l'hypothalamus est parfaitement révélé.

\section{Discussion et conclusion.}

1) La technique proposée permet d'immobiliser la somatostatine de façon irréversible au sein des péricaryons.

Cefte fixation est compatible avec une déshydratation et une inclusion à la paraffine. L'emploi de cette technique présente un certain nombre de difficultés :

a) Un mauvais lavage du lił vasculaire compromet la fixation par formation de précipités embolisants. Ce lavage doit donc être prolongé jusqu'à ce que le liquide ressorte clair. 

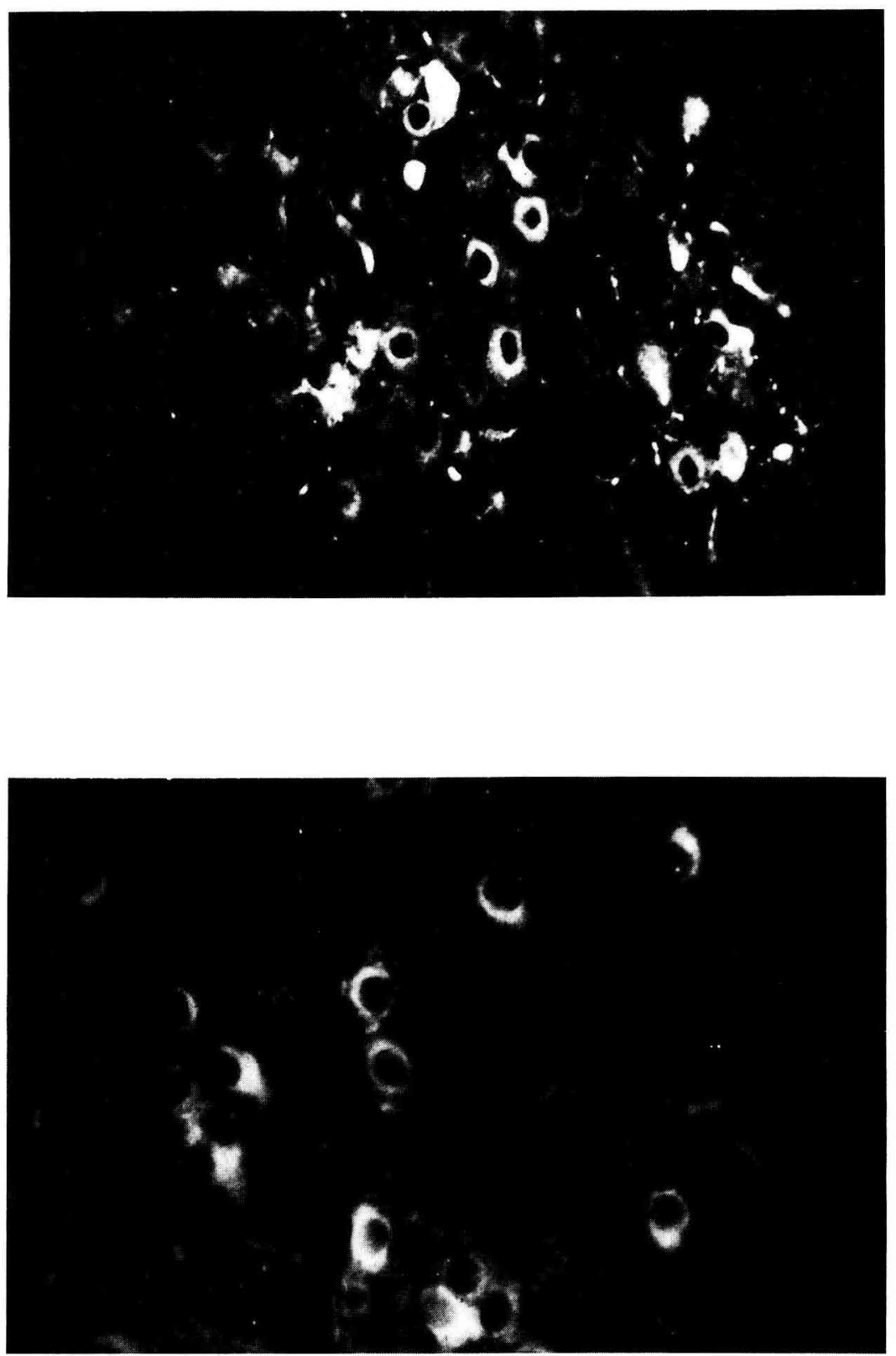

PLANCHE I

Hypothalamus de rat. Fixation (formol-acide tannique-sulfate de cuivre) par perfusion en deux temps.

Immunofluorescence. Sérum anti-somatostatine.

(a) Noyau paraventriculaire. Cellules immunoréactives somatostatine + du système magnocellulaire (G. final : $\times$ 750.) (Réf. : 547-17.)

(b) Zone au contact des parois du recessus supraoptique du $111 \mathrm{l}$ ventricule. Cellules immunoréactives du système parvocellulaire (G. final : $\times 1$ 500.) (Réf. : 548-7.) 
b) La post-fixation formolée à $\mathrm{pH}$ acide entraîne l'apparition d'une fluorescence aspécifique à la périphérie de la coupe lors de la réaction d'immuno-fluorescence. Un lavage prolongé à l'eau courante, après la post-fixation est recommandé ( $24 \mathrm{~h}$ pour un hypothalamus).

c) La durée de perfusion du fixateur doit être limitée à $5 \mathrm{mn}$. Les perfusions dont la durée est supérieure à ce laps de temps compromettent la qualité de la réaction. La comparaison des résultats obtenus avec les deux méthodes préconisées (en un temps et en deux temps), ne laisse pas apparaître de différences évidentes quant à l'intensité de la réaction d'immunofluorescence.

Cependant, la qualité cytologique de la fixation est meilleure après emploi de la méthode de fixation en deux temps.

2) Ces résultals montrent:

- que les fixateurs picroformolés (tels que le Bouin Hollande sublimé), ne permettent pas d'immobiliser le peptide au sein de la totalité des structures qui le contiennent, et de l'y conserver au cours de la déshydratation et de l'inclusion,

- que la méthode de fixation proposée permet la mise en évidence des systèmes péricaryonniques élaborateurs de somatostatine.

L'un d'eux, le système parvocellulaire, a été retrouvé par d'autres auteurs (Hokfelt, 1975 ; Elde et Parsons, 1975 ; Alpert et al., 1976), mais l'existence d'un système magnocellulaire neurophysine positif n'a pas encore été confirmé.

La présence de deux types cellulaires à somatostatine, l'un neurophysine + , l'autre neurophysine -, devra donc être prise en compte dans l'interprétation des résultats immunocytologiques, notamment pour ce qui concerne certaines cellules dont la morphologie ef les dimensions les rapprochent d'éléments du système parvocellulaire, mais dont elles se distinguent par leur caractère neurophysine + .

Acceplé en novembre 1976.

\section{Références}

ALPERT L. C., BRAWER J. R., PATEL Y. C., REICHLIN J., 1976. Somatostatinergic neurons in anterior hypothalamus : Immunohistochemical localization. Endocrinology, 98, 255-259.

DUBOIS M. P., KOLODZIEJCZYK E., 1975. Centres hypothalamiques du rat secrétant la somatostatine : répartition des péricaryons en deux systèmes magno et parvocellulaires (étude immunocytologique). C. R. Acad. Sc., Paris, D, 251, 1737-1740.

DUBOIS M. P., BARRY J., LEONARDELLI J., 1974. Mise en évidence par immunofluorescence et répartition de la somatostatine (SRIF) dans l'éminence médiane des vertébrés (mammifères, oiseaux, amphibiens, poissons). C. R. Acad. Sc., Paris, D, 279, 1899-1903.

ELDE R. P., PARSON J. A., 1975. Immunocytochemical localization of somatostatin in cells bodies of the rat hypothalamus. Amer. J. Anat. 144, 541-548.

HÖKFELT T., EFENDIC S., HELLERSTRÖM C., JOHANSSON O., LUFT R., ARIMURA A., 1975. CelIular localization of somatostatin in endocrine-like cells and neurons, with special reference to the $A_{1}$ cells of the pancreatic islets and the hypothalamus. Acta Endocr. 580, suppl., 200, 5-41.

LEONARDELLI. J., BARRY J., DUBOIS M. P., 1973. Mise en évidence par immunofluorescence d'un constituant immunologiquement apparenté au LH-RF dans l'hypothalamus ef l'éminence médiane chez les mammifères. C. R. Acad. Sc., Paris, D, 276, 2043-2046.

MIZUHIRA V., FUTASAEKU Y., 1972. New fixation for biological membranes using tannic acid. Acta Histochem. Cytochem., 5, 233-235. 